\title{
Targeted overexpression of elafin protects mice against cardiac dysfunction and mortality following viral myocarditis
}

\author{
Syed H.E. Zaidi, ${ }^{1}$ Chi-Chung Hui, ${ }^{2}$ Alexander Y.L. Cheah, ${ }^{1}$ Xiao-Mang You, ${ }^{3}$ \\ Mansoor Husain, ${ }^{3}$ and Marlene Rabinovitch ${ }^{1}$ \\ ${ }^{1}$ Program in Cardiovascular Research, The Hospital for Sick Children, and Departments of Pediatrics, Laboratory Medicine, and \\ Pathobiology and Medicine, \\ ${ }^{2}$ Program in Developmental Biology, The Hospital for Sick Children, and Department of Molecular and Medical Genetics, and \\ ${ }^{3}$ Center for Cardiovascular Research, The Toronto Hospital General Division, and Department of Medicine, \\ University of Toronto, Toronto, Ontario, Canada, M5G 1X8
}

Address correspondence to: Marlene Rabinovitch, Division of Cardiovascular Research, Hospital for Sick Children,

555 University Avenue, Toronto, Ontario, Canada, M5G 1X8. Phone: (416) 813-5918; Fax: (416) 813-7480; E-mail:mr@sickkids.on.ca

Received for publication September 3, 1998, and accepted in revised form February 23, 1999.

\begin{abstract}
Serine elastases degrade elastin, stimulate vascular smooth muscle cell migration and proliferation, and are associated with myocardial damage. To evaluate the impact of elastase inhibition on cardiovascular development and disease, transgenic mice were created in which the mouse preproendothelin-1 promoter was used to target elafin overexpression to the cardiovascular system. To distinguish the transgene from endogenous elafin, constructs were made incorporating a FLAG sequence; the COOH-terminus FLAG-tagged elafin construct produced a stable, functionally active gene product and was used to create transgenic mice. Consistent with endothelin expression, abundant elafin mRNA was observed in transgenic F1 embryos (embryonic day 13.5) and in adult transgenic mice heart, trachea, aorta, kidney, lung, and skin, but not in liver, spleen, and intestine. Functional activity of the transgene was confirmed by heightened myocardial elastase inhibitory activity. No tissue abnormalities were detected by light microscopy or elastin content. However, injection of 10 plaque-forming units (PFU) of encephalomyocarditis virus resulted in death within 11 days in 10 out of 12 nontransgenic mice compared with one out of nine transgenic littermates. This reduced mortality was associated with better cardiac function and less myocardial inflammatory damage. Thus, elafin expression may confer a protective advantage in myocarditis and other inflammatory diseases.
\end{abstract}

J. Clin. Invest. 103:1211-1219 (1999).

\section{Introduction}

Elastase and elastase inhibitors likely play important roles in regulating vasculogenesis (1). Increased serine elastase activity has been documented in a number of clinical (2-4) and experimentally induced cardiovascular diseases (5-11). For example, elevated serine elastase activity has been reported in patients with myocardial infarction and unstable angina (4), peripheral and coronary artery disease (2), and abdominal aortic aneurysm (3). In experimental studies, high elastin turnover (5) is associated with increased expression of an endogenous vascular elastase in the development and progression of pulmonary hypertension (6-8, 12). Moreover, inhibition of elastase activity reduces or prevents the development of pulmonary hypertension and associated changes in the pulmonary arteries $(7,8)$. Elevated serine elastase activity is present in coronary arteries following experimental heart transplant $(10,11)$ and in the myocardium associated with cardiac rejection (11) and murine myocarditis (13). In the murine model of myocarditis, inhibition of serine elastase activity with an orally bioavailable elastase inhibitor reduced inflammation and fibrosis and preserved myocardial function (13). Following heterotopic cardiac transplant in rabbits, coronary artery neointimal formation and myocardial rejection were greatly reduced by intravenous administration of the naturally occurring serine elastase inhibitor elafin (11). Elafin has also been used to prevent myocardial damage in a rat myocardial infarct model (14).

Elafin was originally purified from human skin, bronchial secretions $(15,16)$, and cultured keratinocytes (17). The cDNA encodes for a $12-\mathrm{kD}$ a protein that contains a signal peptide, a transglutaminase substrate domain, and the elafin inhibitory domain (17). It belongs to a complex family of genes with multiple isoforms, some of which are expressed in a tissue-specific manner $(18,19)$. The elafin signal peptide targets the protein to the cell membrane, where it is cleaved. The precursor is secreted and either crosslinked to other proteins via the transglutaminase domain $(17,20,21)$ or cleaved by an unknown enzyme to produce the 6-kDa mature elafin inhibitory protein (17). Both the precursor and mature elafin have been shown to possess elastase inhibitory activities (16). The elafin inhibitory domain contains eight cysteines involved in intra- or intermolecular disulfide bonds, and amino acids ala24 and met 25 have been shown to interact with the active site of serine elastases (22). Elafin selectively inhibits human leukocyte elastase (HLE), porcine pancreatic elastase, and proteinase 3 , but not other serine proteinases such as trypsin, chymotrypsin, plasmin, and cathepsin G (23). Elafin is constitutively expressed in the skin, trachea, and blood vessels $(15,20)$ and appears to be induced in disease $(23,24)$.

An elafin-overexpressing transgenic mouse could be 
useful in addressing the role of serine elastases in vascular development and in cardiovascular diseases in which serine elastase inhibition might be of value. In the present study, we created transgenic mice that overexpress human elafin under the regulation of the preproendothelin-1 promoter, thereby assuring a high level of expression in the cardiovascular system $(25,26)$ and other sites (27-29). Moreover, it could be anticipated that the transgene product would be expressed as early in development as embryonic day 9.5 (E9.5), when endothelin is observed (27). It could also be anticipated that expression would increase with injuries where heightened elastase activity would be expected (23), because endothelin is also upregulated in these conditions $(26,29)$.

To distinguish the transgene product from native elafin, we incorporated a FLAG tag. This tagged elafin was processed correctly by vascular endothelial cells and retained biological activity. The transgenic mouse showed expression of elafin in the cardiovascular system, among other organs, and functional activity was also demonstrated. The phenotype of the mice appeared normal, using microscopic and biochemical assessment of elastin content of tissues. The transgenic mice were, however, protected from the sequelae of encephalomyocarditis (EMC) virus, as evidenced by reduced mortality, decreased myocardial inflammatory damage, and improved function. Thus, this transgenic mouse model may be useful in addressing cardiovascular and other conditions in which heightened elastase activity might contribute to pathogenesis.

\section{Methods}

Addition of $5^{\prime}$ untranslated region to buman elafin $C D N A$. Plasmid pDK6 (kindly provided by J.M. Sallenave, University of Edinburgh, Edinburg, Scotland) contained an RT-PCR fragment of elafin cDNA with only six bases at the $5^{\prime}$ untranslated region (UTR), but a full coding region and $3^{\prime}$ UTR. To include the $5^{\prime}$ UTR, we used the DNA as a template in a PCR reaction with the following forward primers based on the published gene sequence for human elafin (30): E1 (5'-ATAAG AATGC GGCCG CAGGC CAAGC TGGAC TGCAT AAAGA TTGGT ATGGC CTTAG CT-3'), E2 (5'-AGATT GGTAT GGCCT TAGCT CTTAG CCAAA CACCT TCCTG ACACC ATGAG GGCCA GC-3'), and a reverse primer E3 (5'-ACAGT CTAGC GGCCG CTTTA TTGGA AAGTG GATGA GAGA-3'). E1 and E2 overlapping primers contain 67 nucleotides of the $5^{\prime}$ UTR of human elafin. NotI restriction sites were also introduced at the $5^{\prime}$ ends of E1 and E3 to facilitate cloning into the expression vectors. The PCR product was then subcloned into the NotI site of pBluescript SK (pHZ4; Stratagene, La Jolla, California, USA), and the insert was sequenced using the T7, T3, and M13 reverse primers.

Addition of FLAG sequences to human elafin $c D N A$. Different constructs were made that contained FLAG sequences at the $\mathrm{NH}_{2}$ terminus of the signal peptide (pHZ6), $\mathrm{COOH}$-terminus of elafin (pHZ7), or $\mathrm{NH}_{2}$-terminus of mature elafin ( $\mathrm{pHZ8}$ ), i.e., the sequence encoding amino acids distal to the precursor transglutaminase domain. To add FLAG to the $\mathrm{NH}_{2}$-terminus of the signal peptide, PCR was performed using $\mathrm{PHZ} 4$, a plasmid that contained the full-length elafin $\mathrm{CDNA}$, and forward primer E4 (5'-ATAAG AATGC GGCCG CAGGC CAAGC TGGAC TGCAT AAAGA TTG-3'), reverse primer E5 (5'-CTTGT CATCG TCGTC CTTGT AGTCC ATGGT GTCAG GAAGG TGT-3'), forward primer E6 (5'-ACAAG GACGA CGATG ACAAG AGGGC CAGCA GCTTC TTGA-3'), and reverse primer E3. FLAG and
anti-FLAG sequences are underlined. Both PCR products were then mixed and denatured, and the $3^{\prime}$ end of the overlap was extended using deoxyribonucleotide triphosphates (dNTPs) and Taq DNA polymerase, followed by PCR using the flanking E4 forward and E3 reverse primers. The resulting PCR product was then digested with NotI and subcloned into the pBluescript SK (pHZ6).

To add FLAG to the COOH-terminus of mature elafin, PCR was performed using $\mathrm{PHZ} 4$, oligonucleotides $\mathrm{E} 4$, reverse primer E7 (5'-ACAAG GACGA CGATG ACAAG TGAGA GGGAG CCGGT CCT-3'), forward primer E8 (5'-CACTT GTCAT CGTCG TCCTT GTAGT CCTGG GGAAC GAAAC AGGCC$3^{\prime}$ ), and reverse primer E3. Both PCR products were then fused by PCR and subcloned into pBluescript SK as described previously, generating $\mathrm{pHZ7}$.

FLAG was added to the $\mathrm{NH}_{2}$-terminus of mature elafin by PCR using pHZ4 as a template and oligonucleotides E4 and reverse primer E10 (5'-TCGTG TCATC GTCGT CCTTG TAGTC CGCTT TGACT TTATC TTGAC C-3'), and E9(5'-ACAAG GACGA CGATG ACAAG CAAGA GCCAG TCAAA GGTCC-3') and reverse primer E3. Both PCR products were then fused by PCR and subcloned into pBluescript SK, as described above, generating $\mathrm{pHZ} 8$. Plasmid $\mathrm{pHZ6}$, pHZ7, and $\mathrm{pHZ8}$ were sequenced, and correct insertion of FLAG was confirmed.

Generation of preproendothelin-1-directed expression vectors. Elafin cDNA containing inserts were excised from $\mathrm{pHZ} 4$, pHZ6, pHZ7, and $\mathrm{pHZ} 8$ and subcloned into the NotI restriction site of the preproendothelin expression vector pHL3 (p5.9mPPET-LUC described in ref. 25 and kindly provided by Steven Hemmerick, Roche Bio-Sciences, Palo Alto, California, USA) after removing the luciferase insert following NotI digestion. Plasmid pHZ5, pHZ9, pHZ10, and pHZ11, respectively, contained full-length elafin and elafin with a FLAG sequence fused to the $\mathrm{NH}_{2}$-terminus of the signal peptide, the $\mathrm{COOH}$-terminus of elafin, and the $\mathrm{NH}_{2}$-terminus of mature elafin.

Cell culture and transfections. Bovine pulmonary artery endothelial cells (CPAE) were obtained from American Type Culture Collection (Rockville, Maryland, USA). Fetal ovine (100-day gestation) pulmonary artery endothelial cells (LPAE) were harvested, cultured, and characterized as previously described (31). CPAE and LPAE cells were cultured in DMEM and medium 199 (Ontario Cancer Institute, Toronto, Ontario, Canada) containing $10 \% \mathrm{FBS}, 100 \mathrm{U} / \mathrm{ml}$ penicillin, $100 \mu \mathrm{g} / \mathrm{ml}$ streptomycin, and $250 \mathrm{ng} / \mathrm{ml}$ Fungizone. Cells were transfected with calcium phosphate (32) or lipofectamine (GIBCO BRL, Burlington, Ontario, Canada) in different experiments as described in Results. Briefly, $10 \mu \mathrm{g}$ or $3.5 \mu \mathrm{g}$ of DNA was used with $100-\mathrm{mm}$ or $35-\mathrm{mm}$ culture dishes. In some transfection studies, calcium phosphate-precipitated DNA was added to the cells. After four hours, cells were glycerol shocked for $40 \mathrm{~s}$ and kept in serumcontaining medium for $16 \mathrm{~h}$. The medium was then replaced with serum-free medium. For transfection studies using lipofectamine, DNA was mixed with liposomes, and cells were transfected in serum-free medium for four hours followed by culture in $10 \%$ serum-containing medium for $16 \mathrm{~h}$. Depending on the experiment, after $30 \mathrm{~h}$ or five days in serum-free medium, culture medium was collected and analyzed. In cells transfected with the reporter construct (pHL3), extracts were analyzed for luciferase activity (32).

Western immunoblot. Protein extracts from cell culture media were electrophoresed on polyacrylamide Tris-glycine gels and electroblotted to nitrocellulose membranes at $25 \mathrm{~V}$ for 1.5 hours. To block nonspecific binding, membranes were blocked overnight at $4{ }^{\circ} \mathrm{C}$ in $5 \%$ nonfat dry milk in Tris-buffered saline containing $0.1 \%$ Tween- 20 (TBST). Membranes were then incubated for one hour in rabbit anti-human elafin sera $(1: 1,500$; kindly provided by J.M. Sallenave, University of Edinburgh, Edinburgh, Scotland) or M2 monoclonal anti-FLAG $(10 \mu \mathrm{g} / \mathrm{ml})$ 
antibodies (Eastman Kodak, New Haven, Connecticut, USA) diluted in TBST. The membranes were washed in TBST and incubated with goat anti-rabbit IgG or goat anti-mouse IgG (Bio-Rad Laboratories Inc., Hercules, California, USA) peroxidase-conjugated secondary antibodies for one hour, followed by washing in TBST and detection using the enhanced chemiluminescence system (Amersham International Plc., Oakville, Ontario, Canada).

Assay for elafin activity. Elafin's biological activity was assayed in serum-free cell culture medium and in hearts from transgenic mice. Hearts were harvested from mice, washed with saline, and homogenized in water. Extracts were then centrifuged at 15,000 $g$ for $15 \mathrm{~min}$ at $4^{\circ} \mathrm{C}$. The pellet was then washed three times, resuspended in water, boiled for one minute, and recentrifuged; the supernatant was then used in the assay. To ensure that comparisons reflected equal amounts of protein, protein microassays (Bio-Rad Laboratories Inc.) were carried out. Elafin's biological activity was monitored by the inhibition of HLE. Samples were incubated with 4-8 ng of HLE (Elastin Products, Owensville, Missouri, USA) and a fluorescent peptide substrate (20 $\mu \mathrm{M}$ final concentration), AFC091 (MeOSuc-Ala-Ala-AlaAFC; Enzyme Systems Products Inc., Livermore, California, USA). A change in fluorescence, indicating the amount of substrate cleaved, was monitored over a period of $10 \mathrm{~min}$.

Creation of transgenic mice. Plasmid $\mathrm{pHZ10}$ was digested with XhoI, and an $8.5-\mathrm{kb}$ fragment containing the $5.9-\mathrm{kb}$ preproendothelin promoter, COOH-terminus FLAG-tagged elafin, SV40 polyadenylation signal, and endothelin-1 intron was gel-purified using Elutip-D column (Schleicher \& Schuell, Keene, New Hampshire, USA). DNA (1-4 ng/ $\mu \mathrm{l}$ ) was then used for pronuclear injection of eggs from B6/SJL mice obtained from The Jackson Laboratory (Bar Harbor, Maine, USA) and transferred into the oviducts of CD1 foster mothers. Founder mice were identified by dot blot and Southern blot hybridization using tail DNA and ${ }^{32} \mathrm{P}$-radiolabeled human elafin cDNA.

Northern, Southern, and dot blot analyses. Total RNA was isolated from tissues using Trizol (GIBCO BRL) according to the protocol provided by the manufacturer; it was then electrophoresed on formaldehyde agarose gels and transferred to Hybond $\mathrm{N}+$ membranes (Amersham International Plc.) (32). For Southern blot analyses, restriction enzyme-digested DNA was electrophoresed on agarose gels and transferred to Hybond $\mathrm{N}+$ membranes (32). For dot blotting, 5-8 $\mu \mathrm{g}$ of total genomic DNA was blotted onto Hybond $\mathrm{N}+$ membranes using a dot blot apparatus (Bio-Rad Laboratories Inc.) and the method described (32). All membranes were hybridized for one hour with radiolabeled human elafin cDNA and QuikHyb solution (Stratagene, La Jolla, California, USA). Transgene copy number was estimated by comparing the intensity of the hybridization signals in transgenic mice with the known amount of plasmid DNA (0.8-8,000 pg of pHZ10).

Immunostaining. Embryos were fixed overnight in 4\% paraformaldehyde and embedded in paraffin. Sections were taken for histology, deparaffinized using xylene, and gradually rehydrated in ethanol. VectaStain ABC kit (Vector Laboratories, Burlington, Ontario, Canada) was used for immunoperoxidase staining according to the manufacturer's instructions. Rabbit anti-FLAG polyclonal antibodies (Zymed Laboratories, San Francisco, California, USA) $(5 \mu \mathrm{g} / \mathrm{ml}$ final concentration) were used for approximately $16 \mathrm{~h}$ at $4^{\circ} \mathrm{C}$. Immune complexes were then stained by incubation in a solution containing $0.5 \mathrm{mg} / \mathrm{ml}$ solution of 3,3'-diaminobenzidine (Sigma Chemical Co., St. Louis, Missouri, USA) in $50 \mathrm{mM}$ Tris buffer ( $\mathrm{pH} \mathrm{7.4)}$ and $0.015 \%$ hydrogen peroxide. Sections were then counterstained with hematoxylin.

Elastin content in mouse tissues. Measurement of elastin content was carried out as described previously (33) using a Ninhydrin assay (34). Because of tissue mass and density, hearts were homogenized using a Polytron, tissues were centrifuged, and the pellets were resuspended in $0.1 \%$ SDS and boiled twice for $20 \mathrm{~min}$. Pellets from the homogenized and boiled heart tissue samples and intact (nonhomogenized) lung, liver, aorta, and trachea were digested with cyanogen bromide $(50 \mathrm{mg} / \mathrm{ml})$ overnight in $70 \%$ formic acid at room temperature. Elastin residues were then washed twice in boiling water, hydrolyzed overnight in $5.7 \mathrm{~N} \mathrm{HCl}$ at $110^{\circ} \mathrm{C}$, dried, and redissolved in water. Amino acid contents of the hydrolyzed samples were estimated using Ninhydrin reagent (Sigma Chemical Co.). Elastin concentration in the various samples was inferred using a standard plot generated following hydrolysis of known quantities of chicken elastin.

Induction of experimental myocarditis. Mice were injected intraperitoneally with 10 plaque-forming units (PFU) of EMC virus (provided by Peter Liu, Toronto Hospital General Division, Toronto, Ontario, Canada) as in previous studies $(13,35)$, in keeping with a protocol approved by the Animal Care Committee of the Hospital for Sick Children. Consistent with previous studies, the initial protocol was designed to assess the severity of inflammation at $14 \mathrm{~d}$. However, because of an unanticipated high mortality rate with this strain of mice, the protocol was redirected to a second experiment to examine myocardial tissues and function at six days after inoculation of the virus. For functional studies, animals were anesthetized with an intraperitoneal injection of $10 \mathrm{mg} / \mathrm{ml}$ of ketamine (MTC Pharmaceuticals, Cambridge, Ontario, Canada) and 10\% xylazine $(10 \mathrm{mg} / \mathrm{kg})$ (Bayer Inc., Toronto, Ontario, Canada), and the surgical procedure was done under a dissecting microscope (MZ6; Leica, Heerbrugg, Switzerland). The right carotid artery was exposed by making a midline incision on the ventral side of the neck. Two ligatures were placed around the carotid artery, and the distal ligature was tied off. A microcatheter (0.5 $\mathrm{mm}$ diameter) was introduced through an incision between the two ligatures, and the blood pressures were measured in the carotid artery and left ventricle (model 300; Micro-Med, Louisville, Kentucky, USA). Traces were recorded onto a computer using Fura software (developed by P. Backx, University of Toronto) and analyzed using Microcal Origin (Microcal Software Inc., Northampton, Massachusetts, USA). After completion of these studies, the hearts were dissected, rinsed in PBS, fixed overnight in $10 \%$ formalin, embedded in paraffin, sectioned, and stained with hematoxylin and eosin or Movat pentachrome stain. Using a $\times 2$ objective lens, full microscopic cross-sections of each heart were captured directly onto a computer, and images were analyzed by the Image-Pro Plus program (Media Cybernetics, Silver Springs, Maryland, USA). Inflammation was quantified as the amount of inflammatory cell area (percent) per total myocardial area. The damage was considered to be the area containing necrosis, edema, and inflammation and was quantified as a percentage of total myocardial area.

Statistical analysis. All data are presented as mean \pm SEM. Twoway comparisons were made with the Student's $t$ test. $P<0.05$ was considered statistically significant.

\section{Results}

Preproendothelin-1-directed buman elafin expression in pulmonary artery endothelial cells. Plasmids pHZ5 and pHL3, which contained the preproendothelin-directed fulllength human elafin or luciferase genes, respectively (Figure 1a), were transfected into bovine (CPAE) or fetal ovine (LPAE) pulmonary artery endothelial cells (Figure $1 b)$. Immunodetection of human elafin was negative in pulmonary artery endothelial cells transfected with the luciferase plasmid, but transfection with the plasmid containing human elafin under the regulation of the 


\section{Figure 1}

Human elafin expressed by the preproendothelin promoter is processed and secreted by pulmonary artery endothelial cells. (a) Expression vectors contain 5.9-kb mouse preproendothelin-1 promoter, luciferase, or full-length human elafin cDNA, SV40 polyadenylation signals and intron, endothelin-1 (ET-1) intron, and the pBluescript SK (BISK). Positions of signal peptide (SP), transglutaminase (TG) substrate domain, and elafin inhibitory domain are shown at the top. (b) Bovine (CPAE) and 100-day gestation fetal ovine (LPAE) pulmonary artery endothelial cells were transfected with pHL3 (L, luciferase) or pHZ5 ( $E$, human elafin) constructs using calcium phosphate, and culture medium was analyzed by Western immunoblotting using anti-human elafin antisera. Migration of molecular weight markers is denoted along the left. (c) Addition of FLAG epitope to human elafin. Several constructs were generated and contained FLAG sequences at the $\mathrm{NH}_{2}$-terminus of signal peptide ( $\mathrm{pHZ9}), \mathrm{COOH}$-terminus of elafin $(\mathrm{pHZ10})$, and $\mathrm{NH}_{2}$-terminus of mature elafin ( $\mathrm{pHZ11}$ ). These constructs were then evaluated for FLAG-tagged elafin processing in CPAE cells, using the transfection conditions described for the $\mathrm{pHZ} 5$ construct. Anti-FLAG monoclonal antibody M2 was used for the Western immunoblot and demonstrated that the precursor and the processed elafin forms were detectable in culture medium of CPAE cells transfected with $\mathrm{COOH}$-terminus and $\mathrm{NH}_{2}$-terminus FLAGtagged mature elafin constructs. L, 5, 9, 10, and 11, along the top of the Western blot, represent cells transfected with $\mathrm{pHL} 3$ (luciferase), pHZ5, pHZ9, pHZ10, and pHZ11, respectively. Schematic representation of processing, shown on the left, indicates signal peptide cleavage, secretion of precursor, and processing to release mature elafin. a

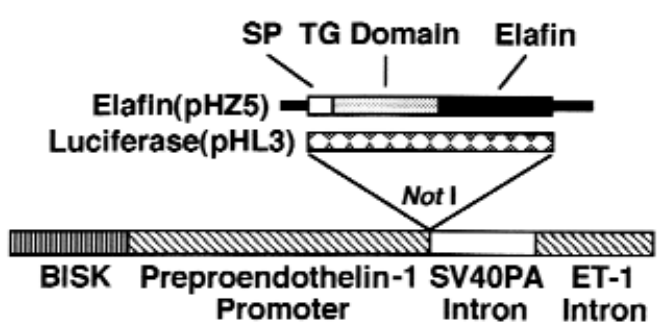

b

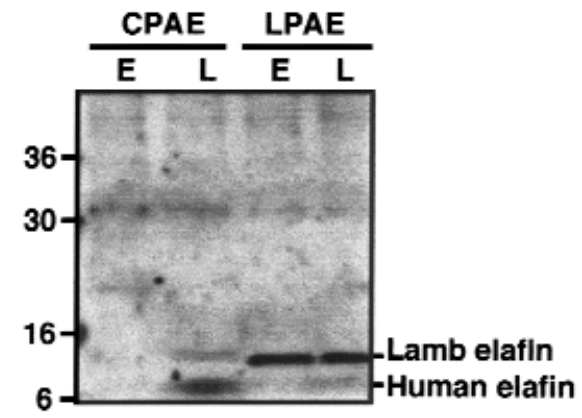

C
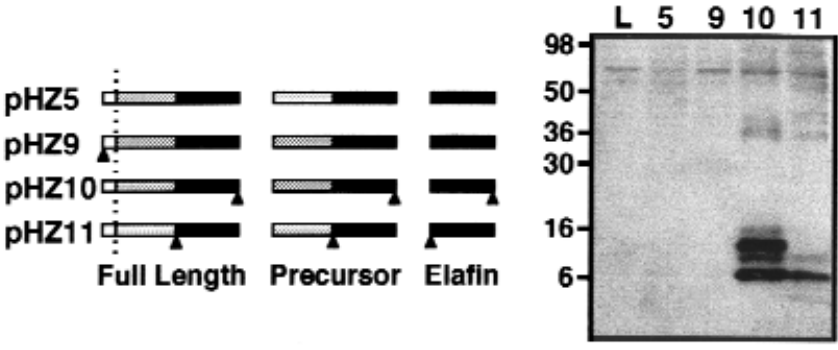

preproendothelin-1 promoter showed that the protein was correctly processed and secreted into the medium. Both the precursor $(\sim 10 \mathrm{kDa})$ and the processed forms of mature elafin $(\sim 6 \mathrm{kDa})$ were evident. Expression of elafin in LPAE cells was much lower when compared with the CPAE cells, as assessed by luciferase activity after cotransfection with the luciferase plasmid PHL3 (data not shown). Also, in LPAE cells, a protein band of $\sim 9 \mathrm{kDa}$ that strongly cross-reacted with the anti-human elafin antibody was apparent in both the human elafinand luciferase-transfected cells, suggesting that ovine elafin was endogenously expressed.

Because of the potential cross-reactivity of anti-human elafin antibodies with endogenous murine elafin, a FLAG epitope sequence (DYKDDDDK) was incorporated into the human elafin cDNA as described in Methods. These constructs, together with plasmid pHL3 containing the luciferase gene and pHZ5 (elafin without FLAG), were transfected into CPAE cells, and culture medium was analyzed for the FLAG-elafin by Western immunoblot using M2 anti-FLAG monoclonal antibodies (Figure 1c). As expected, both the $\mathrm{COOH}$-terminus and the $\mathrm{NH}_{2}$-terminus FLAG-tagged elafin were secreted in the culture medium. The COOH-terminus FLAG-tagged elafin was judged to be more stable than the $\mathrm{NH}_{2}$-terminus FLAG-tagged elafin because it was present in higher concentration in the culture media. Loss of precursor from the $\mathrm{NH}_{2}$-terminus FLAG-tagged elafin likely is due to either rapid processing or degradation of the protein.

Processed COOH-terminus FLAG-tagged elafin (from

\section{Figure 2}

$\mathrm{COOH}$-terminus FLAG-tagged elafin is functionally active. Bovine pulmonary artery endothelial cells were transfected with pHL3 (luciferase), pHZ5 (human elafin without FLAG), or pHZ10 (COOH-terminus FLAG-tagged human elafin) constructs using lipofectamine. Four independent transfections were performed for each construct. Five days after transfection, culture media were analyzed for HLE inhibitory activity as described in Methods. One unit of inhibitory activity is defined as the amount of inhibitor required to reduce $1 \mathrm{ng}$ of HLE activity by $50 \%$. Bars represent mean $\pm \operatorname{SEM}(n=4)$.

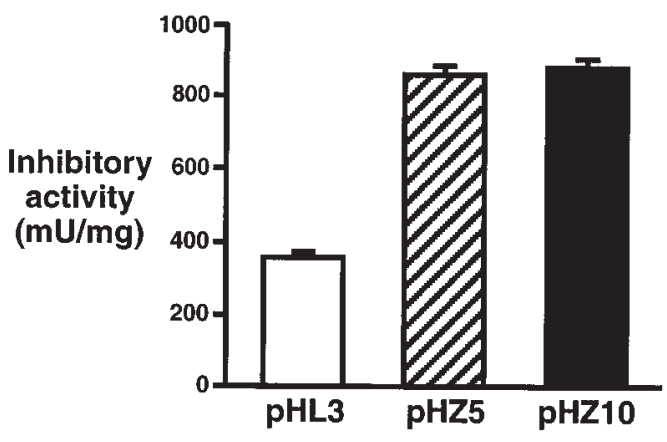


construct pHZ10) was then evaluated for its inhibitory activity against HLE. CPAE cells transfected with plasmid pHZ5 (elafin without FLAG) or pHZ10 showed 2.4 times higher inhibitory activity than those transfected with pHL3 (luciferase) $(P=0.001$; Figure 2). The inhibitory activity of pHZ10-transfected cells was not significantly different from cells transfected with pHZ5. Cells transfected with pHL3 exhibited endogenous inhibitory activity that could be due to elafin or other inhibitors of HLE produced by these cells.

Multiple copies of transgenes are tandemly integrated into the mouse genome. Southern blot of genomic DNA digested with $\mathrm{BamHI}$ from four of the five founders revealed a 4$\mathrm{kb} B a m \mathrm{HI}$ fragment, as expected, suggesting that the transgene is intact and downstream of the preproendothelin-1 promoter (Figure 3a, left). Transgenic lines $2 \mathrm{M}$ and $14 \mathrm{~F}$ had five to 10 copies, $3 \mathrm{M}$ one to two copies, and 7F 10 to 20 copies per genome. To identify the orientation of these multiple copies, genomic DNA was digested with NheI, which has two sites upstream of the elafin cDNA in the transgene (Figure $3 \mathrm{a}$, right). Hybridization with elafin cDNA determines whether integration of multiple copies is tandem, reverse, or scattered (Figure $3 \mathrm{~b}$ ). Transgenic founders 14F, 2M, and 3M showed correct orientation of the transgene. In founder $7 \mathrm{~F}$, most copies were arranged with tandem repeats and two copies in reverse orientation. All founders except $7 \mathrm{~F}$ showed germ-line transmission.

Tissue-specific expression of elafin in transgenic mice. To explore tissue-specific human elafin mRNA expression in transgenic mice, Northern blot analyses were performed on total RNA isolated from an F1 transgenic mouse from the $2 \mathrm{M}$ line (Figure 4). Abundant mRNA expression was observed in the heart, trachea, kidney, aorta, lung, and skin, while liver, spleen, brain, and intestine were negative. Human elafin mRNA was also expressed during development as seen in 13.5-day-old transgenic mouse embryo. No elafin mRNA differences were observed between male or female transgenic mice. Progeny from transgenic line $14 \mathrm{~F}$ showed a similar profile of elafin mRNA expression. Nontransgenic mice showed no hybridization, revealing that human elafin cDNA does not cross-hybridize with the mouse endogenous elafin mRNA. Transgenic and nontransgenic embryos (E13.5), progeny from the $2 \mathrm{M}$ founder, were sectioned sagittally and immunostained with rabbit anti-FLAG polyclonal antibodies. Elafin protein expression was observed in the myocardium, trachea, lung, pulmonary outflow tract, aortic arch, and large blood vessels (Figure 5).

Elastase inhibitory activity is elevated in transgenic mouse hearts. Transgenic and nontransgenic littermates from the $2 \mathrm{M}$ line were analyzed for the presence of elastase inhibitory activity in the heart. This is a minimum estimate and does not account for the elafin released into the blood or cross-linked to extracellular matrix components. Transgenic mice showed more than onefold elevated elastase inhibitory activity compared with nontransgenic mice (Figure 6).

Tissue elastin content. Since no overt differences in morphology of tissues or in elastin were apparent by light microscopy, we confirmed that transgenic mice do not differ from nontransgenic littermates in the elastin con- a

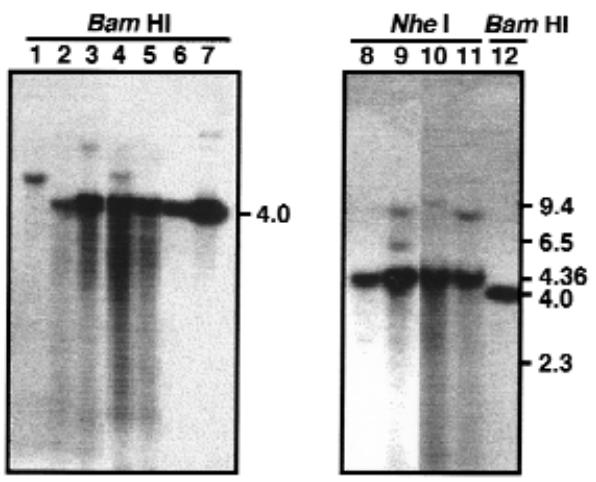

b

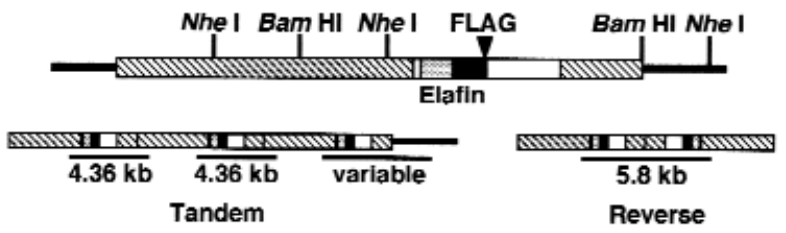

Figure 3

Creation of transgenic mice. (a) DNA isolated from putative transgenic founder mice was digested with $\mathrm{Bam} \mathrm{HI}$ or Nhel, and Southern hybridization was performed using radiolabeled human elafin cDNA. Numbers along the top represent DNA isolated from individual founder mice. Lane 1 (2Ma), lanes 2 and $8(3 \mathrm{M})$, lanes 3 and $9(7 \mathrm{~F})$, lanes 4 and $10(2 \mathrm{M})$, lanes 5 and 11 (4F), and lanes 6, 7, and 12 are $\mathrm{pHZ10}$ digested with $\mathrm{BamHI}$, resulting in a 4-kb fragment containing elafin cDNA. Molecular size markers and the position of 4-kb Bam HI fragments from $\mathrm{pHZ10}$ are shown along the right side of the blots. (b) A schematic representation of the human elafin harboring restriction fragments obtained by Nhel or Bam $\mathrm{HI}$ restriction enzymes is shown. BamHI will always produce a 4-kb fragment if the transgene cassette is intact; 4.36-kb Nhel-generated fragments can only be detected if transgene copies are integrated in a head-to-tail orientation. Integration in a reverse orientation would produce a 5.8-kb Nhel fragment.

a

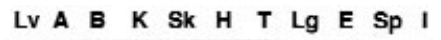

b

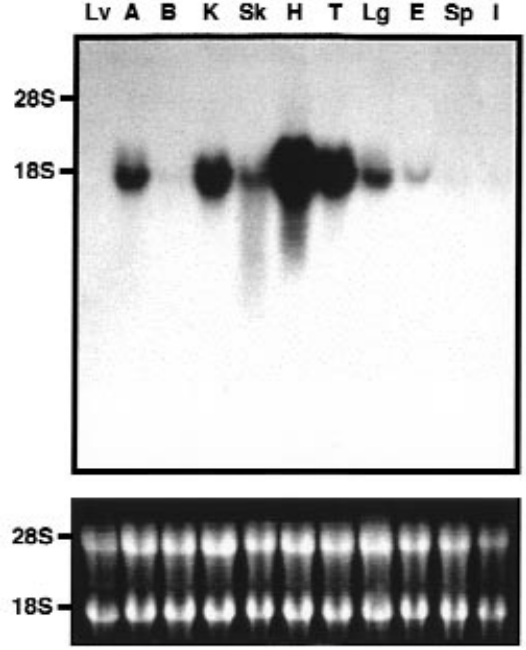

Figure 4

Human elafin mRNA expression in mice. (a) Tissues were harvested from adult (15 weeks old) transgenic F1 mice from 2M founder, and Northern hybridization was performed on total RNAs $(15 \mu \mathrm{g})$ using radiolabeled human elafin cDNA. Liver ( Lv), aorta (A), brain (B), kidney (K), skin (Sk), heart $(H)$, trachea $(T)$, lung $(\mathrm{Lg})$, 13.5-day-old embryo $(E)$, spleen $(S p)$, and intestine (I) are denoted along the top. (b) A photograph of ethidium bromide-stained RNA. Position of 28 and 18 S ribosomal RNAs is denoted along the left. 


\section{Figure 5}

Elafin protein expression in transgenic mouse embryo. Transgenic founder $2 \mathrm{M}$ was cross-bred with wild-type CD1 female. Postfertilization 13.5-day-old embryos were retrieved and genotyped by dot blot hybridization of amniotic sac genomic DNA, using radiolabeled human elafin cDNA. Sagittal sections from transgenic and nontransgenic littermate embryos were stained with rabbit anti-FLAG polyclonal antibodies. All sections were counterstained with hematoxylin stain. $\mathbf{a}, \mathbf{c}$, and $\mathbf{e}$ are from transgenic embryos; $\mathbf{b}, \mathbf{d}$, and $\mathbf{f}$ are from nontransgenic embryos. Cross-sections of pulmonary artery (pa) outflow tract, aortic arch (aa), and trachea (t) are shown in $\mathbf{a}$ and $\mathbf{b}$. $\mathbf{c}$ and $\mathbf{d}$ show ventricular myocardium. Right accessory lobe of lung $(\lg )$ and atria (at) are shown in e and f. Scale bar: $125 \mu \mathrm{m}$.

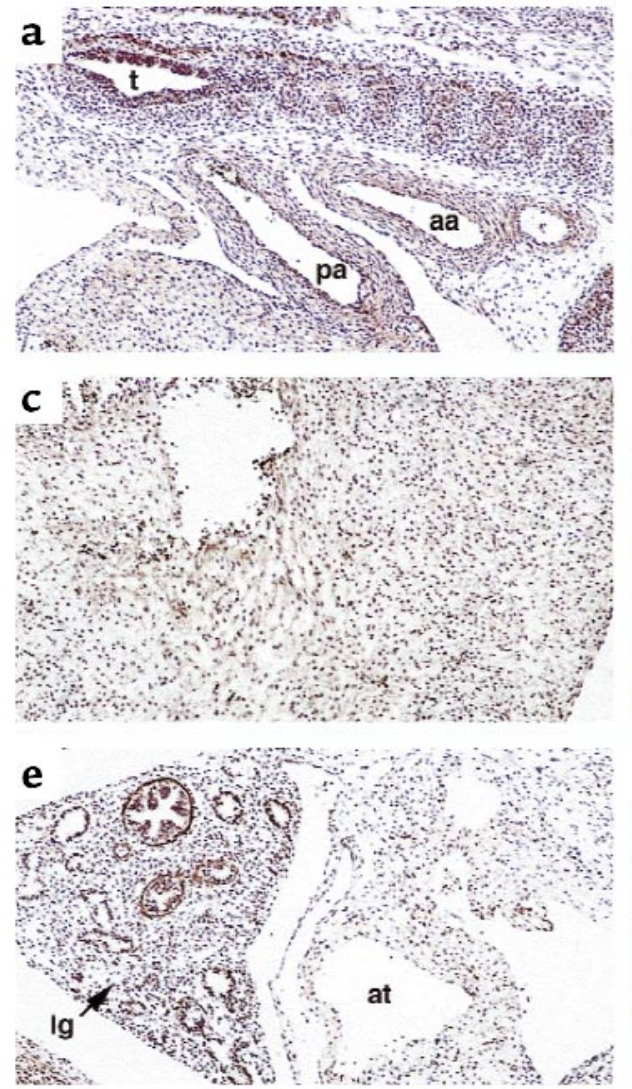

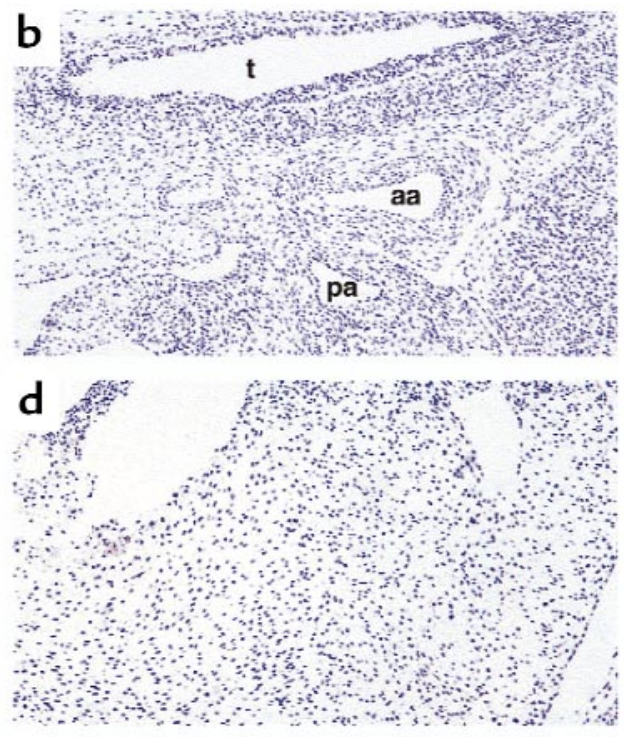

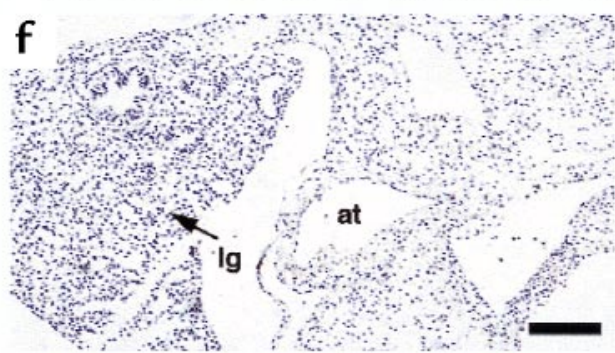

tent of their tissues (Table 1). As a negative control, we used liver that did not express the elafin transgene.

Infection with EMC virus. Progeny from $2 \mathrm{M}$ and $14 \mathrm{~F}$ exhibited similar levels of mRNA expression and tissue distribution, but because of the convenience of generating large numbers of $\mathrm{F} 1$ littermates, the $2 \mathrm{M}$ founder male was used instead of the $14 \mathrm{~F}$ female. To test the importance of elafin overexpression in a cardiac disease model, we assessed EMC virus-induced myocardial damage, which has been well characterized in a DBA/2 strain of mice. Infection with the virus results in inflammation

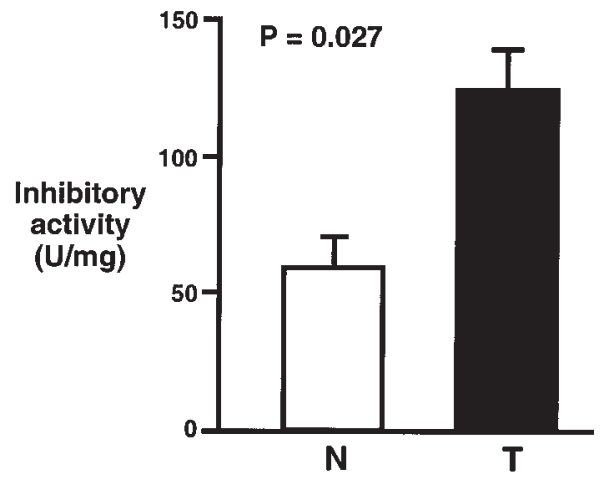

\section{Figure 6}

Transgenic mice hearts exhibit elevated elastase inhibitory activity. Hearts were harvested from two- to four-month-old F1 transgenic $(T)$ and nontransgenic $(\mathrm{N})$ littermates from $2 \mathrm{M}$ transgenic founder and $\mathrm{CD} 1$ wildtype female. Extracts were then used in an assay with HLE as described earlier. Bars represent mean $\pm \operatorname{SEM}(n=3$ animals per group). that reaches a maximum at $14 \mathrm{~d}$, followed by fibrosis and impaired myocardial function evident at $28 \mathrm{~d}$. Within 11 $\mathrm{d}$ of intraperitoneal injection with $10 \mathrm{PFU}$ of EMC virus, 10 out of 12 nontransgenic mice had died, compared with only one out of nine transgenic littermates (Figure 7). None of the mice showed signs of encephalitis on coronal sections of postmortem brains. To confirm that mortality in transgenic mice was related to myocardial damage, we performed another experiment in which we determined whether cardiac function of transgenic and nontransgenic mice were divergent at day 7 after infec- 

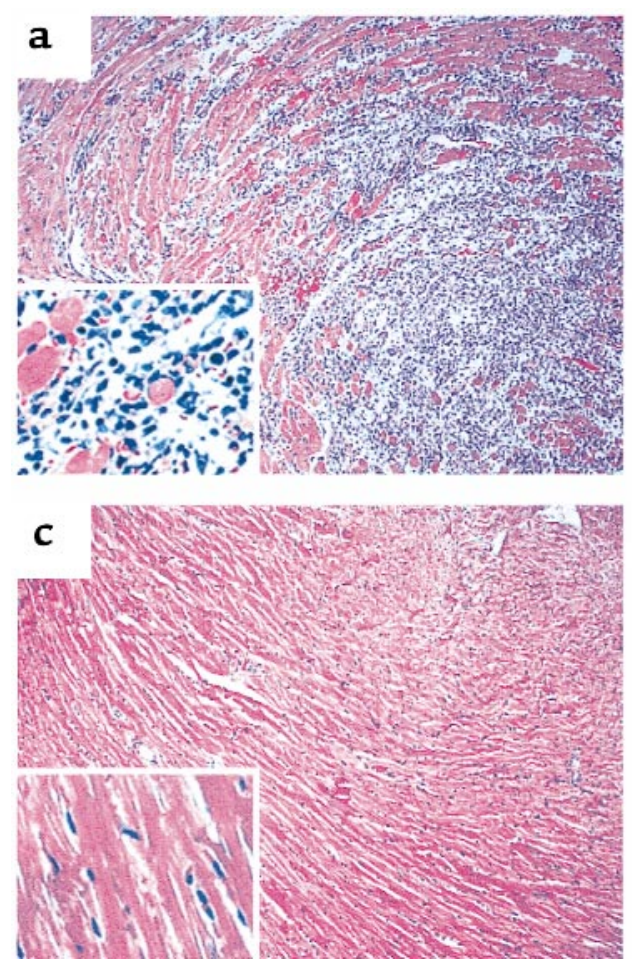

$\mathbf{e}$

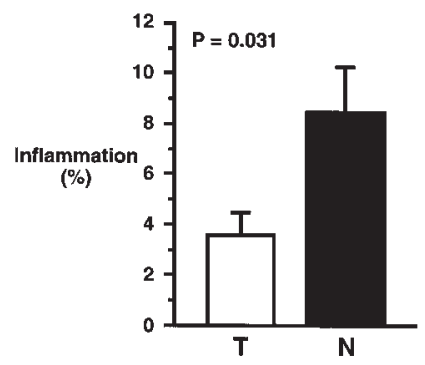

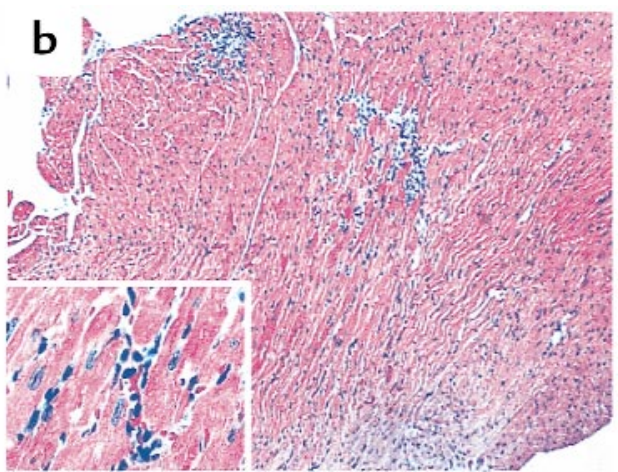

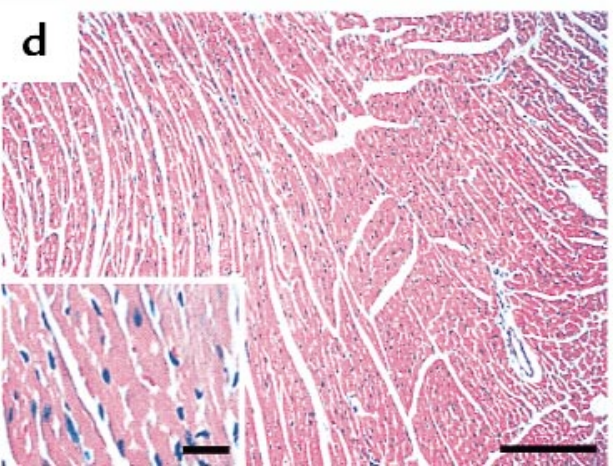

f
Figure 8

Analysis of myocardial inflammation and damage after infection with EMC virus. Hearts from EMCinfected transgenic or nontransgenic littermates were harvested and analyzed as described in Methods. Representative photomicrographs of the myocardium are shown. (a) Nontransgenic infected mice showing a large focus of inflammation and myocyte loss. (b) Transgenic infected mice showing milder inflammation and damage. (c) Nontransgenic noninfected control mice. (d) Transgenic noninfected control mice. Insets show higher magnification of the myocardium. Scale bars: $25 \mu \mathrm{m}$ (insets) and 200 $\mu \mathrm{m}$. Quantification of inflammation and damaged area of the infected myocardium (mean \pm SEM $)$ is shown in $\mathbf{e}$ and $\mathbf{f}(n=12$ for transgenic mice; $n=13$ for nontransgenic littermates). tion and could account for the subsequent increase in mortality in nontransgenic mice (Table 2). Of 12 transgenic and 13 nontransgenic mice infected with $10 \mathrm{PFU}$ of virus, one transgenic and one nontransgenic mouse died during the evaluation at day 7 . Maximum $+\mathrm{dp} / \mathrm{dt}$ values were $\sim 1.8$ times higher in the remaining elafin transgenic mice compared with nontransgenic littermates $(P<0.02)$, and values were similar to those in control uninfected mice. Both left ventricular and systemic systolic pressures were also higher in transgenic compared with nontransgenic mice $(P<0.05$ for both). Mean arterial pressure, diastolic blood pressure, and $-\mathrm{dp} / \mathrm{dt}$ values, although not statistically significantly different, showed a trend toward higher values in transgenic mice, whereas heart rates were similar and left ventricular diastolic pressure similarly elevated when compared with noninfected transgenic and nontransgenic littermates.

Hearts from infected transgenic $(n=12)$ and nontransgenic $(n=13)$ littermates were also analyzed for myocardial damage and inflammation seven days after infection with EMC virus. Both the percent of inflammation and the percent of myocardial damage were reduced by $\geq 50 \%$ in transgenic mice compared with the nontransgenic littermates $(P<0.05$ for both) (Figure 8$)$.

\section{Discussion}

We created transgenic mice that overexpress the serine elastase inhibitor elafin in the cardiovascular system under the regulation of the preproendothelin-1 promoter. We confirmed tandem integration of the elafin transgene, expression of elafin by immunohistochemistry and Northern blot, and functional activity attributable to increased production of elafin. The phenotype of the mice was normal, but following inoculation with EMC virus, the cardiac function was preserved and inflammation and damage to myocardium was reduced, resulting in decreased mortality.

Elafin expression in the human has been described in a variety of tissues, including lung, trachea, skin, and blood vessels $(16,20,23)$. In blood vessels, moderate elafin immunostaining was reported in smooth muscle cells (20). To our knowledge, ours is the first report of elafin expression in pulmonary artery endothelial cells. Previous studies have not shown elafin expression in kidney, brain, liver, or heart. Preproendothelin-1 has been more extensively studied and documented in vascular endothelial cells, as well as smooth muscle cells, heart, lung, kidney, brain, and skin (27-29). Increased endothelin expression has been reported in pulmonary hyper- 
Table 1

Elastin contents in various tissues of transgenic and nontransgenic mice

\begin{tabular}{lcc}
\hline Tissue & Transgenic & Nontransgenic \\
Heart & $0.476 \pm .04$ & $0.492 \pm .025$ \\
Aorta & $10.345 \pm .992$ & $12.746 \pm 0.357$ \\
Lung & $2.645 \pm 0.218$ & $2.692 \pm 0.154$ \\
Trachea & $3.122 \pm 0.287$ & $3.272 \pm 0.24$ \\
Liver & $7.125 \pm 1.446$ & $6.916 \pm 1.313$
\end{tabular}

All values are expressed as milligram of elastin per 100 milligrams of wet tissue weight, except lung and liver, where values were normalized to dry weight. Transgenic and nontransgenic four-month-old female littermates ( $n=5$ in each group) were used for this study. All samples were analyzed in triplicate.

\section{Table 2}

Analysis of cardiac function in mice subjected to ECM virus infection

\begin{tabular}{lccc}
\hline & & & \\
Parameters & Transgenic & Nontransgenic & $P$ value \\
$+\mathrm{dp} / \mathrm{dt}$ & $4,472 \pm 533^{\mathrm{A}}$ & $2,519 \pm 544$ & 0.018 \\
& $(4,493 \pm 619)$ & $(4,450 \pm 815)$ & $(0.970)$ \\
-dp/dt & $3,236 \pm 334$ & $2,424 \pm 553$ & 0.233 \\
& $(4,363 \pm 251)$ & $(3,615 \pm 725)$ & $(0.562)$ \\
LVPSP $(\mathrm{mmHg})$ & $101 \pm 4.2^{\mathrm{A}}$ & $73.2 \pm 7.6$ & 0.006 \\
& $(110 \pm 4.4)$ & $(104 \pm 7.5)$ & $(0.537)$ \\
LVEDP $(\mathrm{mmHg})$ & $19.5 \pm 4.1$ & $20.8 \pm 2.8$ & 0.795 \\
& $(5.3 \pm 4.4)$ & $(9.7 \pm 3.4)$ & $(0.455)$ \\
HR (beats per minute) & $270 \pm 24$ & $296 \pm 20$ & 0.413 \\
& $(262 \pm 56)$ & $(267 \pm 21)$ & $(0.930)$ \\
SBP (mmHg) & $110 \pm 6.3^{\mathrm{A}}$ & $87 \pm 7.9$ & 0.036 \\
& $(131 \pm 8.7)$ & $(128 \pm 11)$ & $(0.881)$ \\
DBP $(\mathrm{mmHg})$ & $80.3 \pm 4.9$ & $65.7 \pm 6.4$ & 0.090 \\
& $(81 \pm 8.6)$ & $(83 \pm 3.5)$ & $(0.769)$ \\
MAP $(\mathrm{mmHg})$ & $90.2 \pm 5.2$ & $72.9 \pm 6.9$ & 0.062 \\
& $(97.7 \pm 8.3)$ & $(98.3 \pm 5.8)$ & $(0.949)$
\end{tabular}

Data from control noninfected mice are shown in parentheses. ${ }^{A}$ Significantly different. $\mathrm{DBP}$, diastolic blood pressure; dp/dt, developed pressure over time; HR, heart rate; LVEDP, left ventricular end diastolic pressure; LVPSP, left ventricular peak systolic pressure; MAP, mean arterial pressure; SBP, systolic blood pressure.

tension (36), myocardial ischemia (37), and congestive heart failure (38). These are conditions associated with elevated elastase activity where it would be advantageous to assess the possible protective effects of very high levels of elafin expression. These could likely be better achieved with the preproendothelin-1 than with the elafin promoter. For example, preproendothelin-1-driven luciferase resulted in consistent upregulation of transgene expression during hypoxia in all tissues, especially in the lung (26).

The preproendothelin-1 promoter-luciferase transgenic mouse was used to document that targeted expression to the cardiovascular system could be achieved (25, 26). Endothelin-1 mRNA is observed by in situ hybridization during embryonic development as early as E9.5 (27) in the cardiovascular system, as well as in the pulmonary parenchyma and blood vessels (E13.5). A similar elafin expression pattern was documented in our study in F1 transgenic embryos (E13.5). In adult transgenic mice, human elafin mRNA is also observed by Northern blot analysis in tissues where endothelin is expressed, e.g., heart, aorta, trachea, skin, lung, and kidney. Thus, we documented that elafin is overexpressed early in devel- opment and persists in cardiovascular and other tissues. Elafin overexpression assessed in adult heart tissue manifest as serine elastase inhibitory activity is elevated more than onefold. While this might have interfered with cardiovascular development in the transgenic mice, no morphological differences were apparent by light microscopy, nor were there differences in total elastin content measured biochemically. This would seem to indicate that gain of elafin function of the magnitude achieved is relatively unimportant in vascular development. Heightened elastase could compensate for the increased inhibitory activity, or an increase in elafin early in development may result in a decrease in another inhibitor, e.g., secretory leukocyte protease inhibitor (39).

These results did not exclude the potential significance of overexpression of elafin in cardiovascular diseases. We have shown that elafin protects against the coronary arteriopathy and myocardial necrosis that occurs after experimental heart transplant in rabbits (11). A selective orally bioavailable serine elastase inhibitor protects against cardiac dysfunction and fibrosis following infection of mice with the EMC virus (13). For expediency, we decided to test the efficacy of our model with respect to EMC. Since both the precursor containing the transglutaminase motif and the mature elafin with the inhibitory domain are capable of inhibiting serine elastases, natural overexpression of the gene product may be far preferable to the administration of the mature elafin as was done in previous studies (11). The transglutaminase substrate domain has been shown to covalently crosslink elafin to other proteins and may thereby anchor elafin to its site of action $(17,20,21)$. An additional advantage of using the transgenic mouse to explore the importance of elastase inhibitory function in cardiovascular disease is that delivery of the transgene product to the tissue of interest could be ensured.

Different strains of mice are differentially susceptible to EMC virus (unpublished data from our laboratory), and the genetic background of the animals used in this study (B6/SJL/CD1) resulted in a much more severe form of EMC virus-induced myocarditis than previously reported in $\mathrm{DBA} / 2$ mice $(13,35)$. This more severe form of myocarditis allowed us to show a substantial impact of elafin transgene expression on EMC virus-induced mortality. This impact suggested that serine elastases were not only important in the development of fibrosis, as deduced from our previous work, but were also involved in the initial response of the organism to viral toxicity. Serine elastase inhibitors do not affect EMC virus propagation $(13,40)$. Rather, the protective effect was associated with preserved cardiac function at a time in the evolution of the disease where there is only an early influx of inflammatory cells. There was a significant difference in $+\mathrm{dp} / \mathrm{dt}$ in transgenic compared with nontransgenic mice, suggesting that cardiac function was preserved. We confirmed reduced inflammation and associated myocardial tissue damage.

Since elafin expression has been documented in the vessel wall, agents that increase its level may provide protection against cardiovascular diseases where serine elastases are important. It is possible that endogenous expression of elafin might be a genetic background fac- 
tor that influences susceptibility to disease and explains, for example, why patients infected with the same myocarditis-causing virus can have major differences in initial presentation and recovery.

\section{Acknowledgments}

The authors wish to thank the transgenic facility at the Hospital for Sick Children; Claire Coulber, Sorana Ciura, and Eva Sitarz for technical assistance; and Joan Jowlabar, Judy Edwards, and Judy Matthews for secretarial assistance. This study was supported by Heart and Stroke Foundation of Canada grant HSFO 2649. M. Rabinovitch is a chair of the Heart and Stroke Foundation of Canada, and M. Husain is a Clinician Scientist of the Medical Research Council of Canada.

1. Wong, L.C., and Langille, B.L. 1996. Developmental remodeling of the internal elastic lamina of rabbit arteries. Circ. Res. 78:799-805.

2. Fowkes, F.G., et al. 1993. Cross-linked fibrin degradation products, progression of peripheral arterial disease, and risk of coronary heart disease. Lancet. 342:84-86.

3. Cohen, J.R., Mandell, C., and Wise, L. 1987. Characterization of human aortic elastase found in patients with abdominal aortic aneurysms. Surg. Gynecol. Obstet. 165:301-304.

4. Dinerman, J.L., et al. 1990. Increased neutrophil elastase release in unstable angina pectoris and acute myocardial infarction. J. Am. Coll. Cardiol. 15:1559-1563.

5. Todorovich-Hunter, L., Johnson, D.J., Ranger, P., Keeley, F.W., and Rabinovitch, M. 1988. Altered elastin and collagen synthesis associated with progressive pulmonary hypertension induced by monocrotaline. A biochemical and ultra structural study. Lab. Invest. 58:184-195.

6. Todorovich-Hunter, L., et al. 1992. Increased pulmonary artery elastolytic activity in adult rats with monocrotaline-induced progressive hypertensive pulmonary vascular disease compared with infant rats with non progressive disease. Am. Rev. Respir. Dis. 146:213-223.

7. Maruyama, K., et al. 1991. Chronic hypoxic pulmonary hypertension in rats and increased elastolytic activity. Am. J. Physiol. 261:H1716-H1726.

8. Ye, C.L., and Rabinovitch, M. 1991. Inhibition of elastolysis by SC-37698 reduces development and progression of monocrotaline pulmonary hypertension. Am. J. Physiol. 261:H1255-H1267.

9. Ilkiw, R., Todorovich-Hunter, L., Maruyama, K., Shin, J., and Rabinovitch, M. 1989. SC-39026, a serine elastase inhibitor, prevents muscularization of peripheral arteries, suggesting a mechanism of monocrotaline-induced pulmonary hypertension in rats. Circ. Res. 64:814-822.

10. Oho, S., and Rabinovitch, M. 1994. Post-cardiac transplant arteriopathy in piglets is associated with fragmentation of elastin and increased activity of a serine elastase. Am. J. Pathol. 145:202-210.

11. Cowan, B., et al. 1996. Elafin, a serine elastase inhibitor, attenuates postcardiac transplant coronary arteriopathy and reduces myocardial necrosis in rabbits after heterotopic cardiac transplantation. J. Clin. Invest. 97:2452-2468.

12. Zhu, L., et al. 1994. The endogenous vascular elastase that governs development and progression of monocrotaline-induced pulmonary hyper tension in rats is a novel enzyme related to the serine protease adipsin. $J$. Clin. Invest. 94:1163-1171.

13. Lee, J.K., et al. 1998. Inhibition of serine elastase activity reduces inflammation and fibrosis and preserves cardiac function following experimental murine myocarditis. Nat. Med. 4:1363-1391.

14. Tiefenbacher, C.P., et al. 1997. Inhibition of elastase improves myocardial function after repetitive ischemia and myocardial infarction in the rat heart. Eur. J. Physiol. 433:563-570.

15. Wiedow, O., Schröder, J.-M., Gregory, H., Young, J.A., and Christophers, E. 1990. Elafin: an elastase-specific inhibitor of human skin. J. Biol. Chem. 265:14791-14795.

16. Sallenave, J.-M., and Silva, A. 1993. Characterization and gene sequence of the precursor of elafin, an elastase-specific inhibitor in bronchial secretions. Am. J. Respir. Cell Mol. Biol. 8:439-445.

17. Molhuizen, H.O.F., et al. 1993. SKALP/Elafin: an elastase inhibitor from cultured human keratinocytes. J. Biol. Chem. 268:12028-12032.
18. Zeewen, P., Hendriks, W., de Jong, W.W., and Schalkwijk, J. 1997. Identification and sequence analysis of two new members of the SKALP/elafin and SPAI-2 gene family. J. Biol. Chem. 272:20471-20478.

19. Tamechika, I., et al. 1996. Accelerated evolution in inhibitor domains of porcine elafin family members. J. Biol. Chem. 271:7012-7018.

20. Nara, K., et al. 1994. Elastase inhibitor elafin is a new type of proteinase inhibitor which has a transglutaminase-mediated anchoring sequence termed "cementoin." J. Biochem. 115:441-448.

21. Steinert, P.M., and Marekov, L.N. 1995. The proteins elafin, fillagrin, keratin intermediate filaments, loricrin, and small proline-rich proteins 1 and 2 are isodipeptide cross-linked components of the human epidermal cornified cell envelope. J. Biol. Chem. 270:17702-17711.

22. Tsunemi, M., Matsuura, Y., Sakakibara, S., and Katsube, Y. 1996. Crystal structure of an elastase-specific inhibitor elafin complexed with porcine pancreatic elastase determined at $1.9 \AA$ resolution. Biochemistry. 35:11570-11576

23. Molhuizen, H.O.F., and Schalkwijk, J. 1995. Structural, biochemical, and cell biological aspects of the serine proteinase inhibitor SKALP/Elafin/ESI. Biol. Chem. Hoppe Seyler. 376:1-7.

24. Schalkwijk, J., van Vlijmen, I.M.J.J., Alkemade, J.A.C., and de Jongh, G.J. 1992. Immunohistochemical localization of SKALP/Elafin in psoriatic epidermis. J. Invest. Dermatol. 100:390-393.

25. Harats D., et al. 1995. Targeting gene expression to the vascular wall in transgenic mice using the murine preproendothelin-1 promoter. J. Clin. Invest. 95:1335-1344.

26. Aversa, C.R., et al. 1997. Hypoxia stimulates human preproendothelin-1 promoter activity in transgenic mice. Am. J. Physiol. 273:L848-L855.

27. Chan, T.S.K., Lin, C.X.F., Chan, W.Y., Chung, S.S.M., and Chung, S.K. 1995. Mouse preproendothelin-1 gene: cDNA cloning, sequence analysis and determination of sites of expression during embryonic development. Eur. J. Biochem. 234:819-826.

28. Firth, J.D., and Ratcliffe, P.J. 1992. Organ distribution of three rat endothelin messenger RNAs and the effects of ischemia on renal gene expression. J. Clin. Invest. 90:1023-1031.

29. Rubanyi, G.M., and Botelho, L.H.P. 1991. Endothelins. FASEB J. 5:2713-2720.

30. Saheki, T., et al. 1992. Primary structure of the human elafin precursor preproelafin deduced from the nucleotide sequence of its gene and the presence of unique repetitive sequences in the prosegment. Biochem. Biophys. Res. Commun. 185:240-245.

31. Rabinovitch. M., Beharry, S., Bothwell, T., and Jackowski, G. 1988. Qualitative and quantitative differences in protein synthesis comparing fetal lab ductus arteriosus endothelium and smooth muscle with cells from adjacent vascular sites. Dev. Biol. 130:250-258.

32. Sambrook, J.E., Fritsch, F., and Maniatis, T. 1989. Molecular cloning: a laboratory manual. 2nd edition. Cold Spring Harbor Laboratory Press. Cold Spring Harbor, NY

33. Keeley, F.W., and Alatawi, A. 1991. Response of aortic elastin synthesis and accumulation to developing hypertension and the inhibitory effect of colchicine on this response. Lab. Invest. 64:499-507.

34. Moore, S. 1968. Amino acid analysis: aqueous dimethyl sulfoxide as solvent for the Ninhydrin reaction. J. Biol. Chem. 243:6281-6283.

35. Dong, R., Liu, P., Wee, L., Butany, J., and Sole, M.J. 1992. Verapamil ameliorates the clinical and pathological course of murine myocarditis. $J$. Clin. Invest. 90:2022-2030.

36. Li, H., Elton, S., Chen, Y.F., and Oparil, S. 1994. Increased endothelin receptor gene expression in hypoxic rat lung. Am. J. Physiol. 266:L553-L560.

37. Ezra, D., Goldstein, R.E., Czaja, J.F., and Feuerstein, G.Z. 1989. Lethal ischemia due to intracoronary endothelin in pigs. Am. J. Physiol. 257:H339-H343.

38. Fukuchi, M., and Giaid, A. 1998. Expression of endothelin-1 and endothelin-converting enzyme-1 mRNAs and proteins in failing human hearts. J. Cardiovasc. Pharmacol. 31(Suppl. 1):S421-S423.

39. Sallenave, J.M., Shulmann, J., Crossley, J., Jordana, M., and Gauldie, J. 1994. Regulation of secretory leukocyte proteinase inhibitor (SLPI) and elastase-specific inhibitor (ESI/Elafin) in human airway epithelial cells by cytokines and neutrophilic enzymes. Am. J. Respir. Cell Mol. Biol. 11:733-741.

40. Molla, A., Hellen, C.U.T., and Wimmer, E. 1993. Inhibition of proteolytic activity of poliovirus and rhinovirus $2 \mathrm{~A}$ proteinases by elastase-specific inhibitors. J. Virol. 67:4688-4695. 